\title{
Detection of green apples in natural scenes based on saliency theory and Gaussian curve fitting
}

\author{
Bairong $\mathrm{Li}^{1,2,3}$, Yan Long ${ }^{1,2,3}$, Huaibo Song ${ }^{1,2,3^{*}}$ \\ (1. College of Mechanical and Electronic Engineering, Northwest A\&F University, Yangling 712100, Shaanxi, China; \\ 2. Key Laboratory of Agricultural Internet of Things, Ministry of Agriculture, Yangling 712100, Shaanxi, China; \\ 3. Key Laboratory of Agricultural Information Perception and Intelligent Services, Yangling 712100, Shaanxi, China)
}

\begin{abstract}
Green apple targets are difficult to identify for having similar color with backgrounds such as leaves. The primary goal of this study was to detect green apples in natural scenes by applying saliency detection and Gaussian curve fitting algorithm. Firstly, the image was represented as a close-loop graph with superpixels as nodes. These nodes were ranked based on the similarity to background and foreground queries to generate the final saliency map. Secondly, Gaussian curve fitting was carried out to fit the $V$-component in YUV color space in salient areas, and a threshold was selected to binarize the image. To verify the validity of the proposed algorithm, 55 images were selected and compared with the common used image segmentation algorithms such as $k$-means clustering algorithm and FCM (Fuzzy C-means clustering algorithm). Four parameters including recognition ratio, FPR (false positive rate), FNR (false negative rate) and FDR (false detection rate) were used to evaluate the results, which were $91.84 \%, 1.36 \%, 8.16 \%$ and $4.22 \%$, respectively. The results indicated that it was effective and feasible to apply this method to the detection of green apples in nature scenes.
\end{abstract}

Keywords: image processing, green apple, natural scene, machine vision, object detection, saliency theory, Gaussian curve fitting

DOI: $10.25165 /$ j.ijabe.20181101.2899

Citation: Li B R, Long Y, Song H B. Detection of green apples in natural scenes based on saliency theory and Gaussian curve fitting. Int J Agric \& Biol Eng, 2018; 11(1): 192-198.

\section{Introduction}

It is an important issue to apply machine vision technology in automatic apple target recognition. And it also can be applied in the precision agriculture research fields for production estimation and growth condition monitoring. The application is of great importance for optimizing the management and use of water, fertilizer and pesticides required in apple plants. Because apples are always green before harvest and green apple varieties are promoting, such as the Australian Green Apple and Green Delicious Apple, the green fruit target recognition has received extensive attention of researchers.

At present, many experts and their research teams focused on color combined with texture feature method ${ }^{[1]}$, clustering algorithm ${ }^{[2,3]}$, neural network algorithm ${ }^{[4]}$, clustering algorithm combined with neural network method ${ }^{[5]}$, clustering algorithm combined with homomorphic filtering method ${ }^{[6]}$, evolutionary algorithm $^{[7]}$ and tried to solve the fruit image segmentation problems. However, segmentation problem of green fruit images with complex background cannot be alleviated by these methods.

Previous studies showed that the visual attention mechanism of eyes can guide us to find the most attractive and important areas effectively in complex scenes. It had been applied to numerous

\section{Received date: 2016-10-08 Accepted date: 2017-03-06}

Biographies: Bairong Li, Bachelor, research interests: intelligent detecting, Email: lbairong@163.com; Yan Long, PhD, Lecturer, research interests: digital image processing, Email: longyan@nwsuaf.edu.cn.

*Corresponding author: Huaibo Song, $\mathrm{PhD}$, Associate Professor, research interests: image processing. College of Mechanical and Electronic Engineering, Northwest A\&F University, 22 Xinong Road, Yangling 712100, Shaanxi, China. Tel: +86-29-87092391, Fax: +86-29-87091737, Email: songhuaibo@nwsuaf. edu.cn. vision problems including image segmentation ${ }^{[8]}$, object recognition ${ }^{[9]}$, image compression ${ }^{[10]}$, image retrieval ${ }^{[11]}$, etc. Liu et al. ${ }^{[12]}$ proposed a binary saliency estimation model by training a conditional random field and combining a set of novel features. Wang et al. ${ }^{[13,14]}$ analyzed multiple cues in a unified energy minimization framework and used a graph-based saliency model to detect salient objects. Lu et al. ${ }^{[15]}$ developed a hierarchical graph model and utilized concavity context to compute weights among nodes, from which the graph was bi-partitioned for salient object detection. Cheng et al. ${ }^{[16]}$ considered the global region contrast with respect to the entire image and spatial relationships across the regions to extract saliency map. Xie et al. ${ }^{[17]}$ proposed a novel model for bottom-up saliency within the Bayesian framework by exploiting low and mid level cues. Sun et al. ${ }^{[14]}$ improved Xie's model by introducing boundary and soft segmentation. Background often presents local or global appearance connectivity with each of four image boundaries and foreground presents appearance coherence and consistency.

The objective of the research was to implement a robust and efficient recognition of green fruits, therefore these cues were exploited to compute pixel saliency based on the ranking of superpixels ${ }^{[18]}$. The graph-based manifold ranking algorithm was applied to the saliency detection of green apples. Combined with Gaussian curving fitting, the gray threshold was selected to implement the accurate segmentation of this type of objects.

\section{Materials and methods}

\subsection{Materials}

The Green Delicious Apple was selected as the research object in this study, which is one of the most popular apple cultivars in China. Fifty-five images of apples in natural scenes were tested. All the images were captured by a camera of Fuji film A900, in the 
orchard affiliated to College of Horticulture, Northwest A\&F University, in sunny days of August 2014. They were saved in JPEG format and RGB color model, and their sizes were $1024 \times 768$ pixels. All the programs were developed by using Matlab R2009a in a PC with $2.60 \mathrm{GHz}$ processor and 4.0 GB RAM.

\subsection{Methods}

2.2.1 Segmentation of apple image using clustering algorithm

(1) $k$-means clustering algorithm

The $k$-means algorithm is a well-known partitioning method for clustering. It takes $k$ as input parameter and partitions a set of $n$ objects into $k$ clusters based on their closeness to each other according to the Euclidean distance ${ }^{[19]}$. In this study, $k=3$ was used for the image which was seen as fruit region, leave region and other (branch, sky or grass) regions.

(2) FCM clustering algorithm

FCM was proposed due to its accuracy in segmentation with the presence of intensity inhomogeneity. Pixels were grouped on the basis of membership assigned to pixel according to the Euclidean distance between cluster center and pixel ${ }^{[20]}$. The parameter $C$ corresponded to the number of data set, set $C=3$ as we had done in the $k$-means clustering method.

2.2.2 Saliency detection of green apples via graph-based manifold ranking

Saliency detection via graph-based manifold ranking is a bottom-up method to detect salient regions in images. The saliency of the image elements (superpixels) is defined based on their relevance to the given seeds or queries. In this research, the relevance was computed by ranking the similarity of the image elements with foreground or background cues via graph-based manifold ranking. It was carried out in a two-stage scheme to implement the saliency detection of images efficiently.

(1) Graph-based manifold ranking

The graph-based ranking problem was described as follows:

Given a node as a query, the remaining nodes were ranked based on their relevance to the given query. The goal was to learn a ranking function, which defined the relevance between unlabeled nodes and queries. The main algorithm was Manifold Ranking whose idea was described as follows:

The features of captured images were extracted respectively to develop a high dimensional space of image feature.

1) Given a captured image set $\boldsymbol{I}=\left\{I_{1}, \ldots I_{n}\right\}$ and the feature space of image dataset $\boldsymbol{X}=\left\{x_{1}, \ldots x_{1}, x_{l+1}, \ldots, x_{n}\right\} \in \boldsymbol{R}^{m^{*} n}$, some data points were labeled queries and the rest was needed to be ranked according to their relevance to the queries.

2) Let $d: \boldsymbol{X} \times \boldsymbol{X} \rightarrow \boldsymbol{R}$ represent a measure which defines the Euclidean distance, written as $d\left(x_{i}, x_{j}\right)$, between $x_{i}$ and $x_{j}$.

3) Let $f: \boldsymbol{X} \rightarrow \boldsymbol{R}^{n}$ denote a ranking function which assigns a ranking value $f_{i}$ to each point $x_{i}$, and $f$ can be viewed as a vector $\boldsymbol{f}=$ $\left[f_{l}, \ldots, f_{n}\right]^{\mathrm{T}}$. Let $\boldsymbol{y}=\left[y_{1}, \ldots, y_{n}\right]^{\mathrm{T}}$ denote an indicator vector, in which $y_{i}=1$ if $x_{i}$ was a query, and $y_{i}=0$ otherwise.

4) Define a graph $\boldsymbol{G}=(\boldsymbol{V}, \boldsymbol{E})$ on the dataset, where the nodes $\boldsymbol{V}$ were the dataset $\boldsymbol{X}$ and the edges $\boldsymbol{E}$ were weighted by an affinity matrix $\boldsymbol{W}=\left[w_{i j}\right]_{n \times n}$. The weight was computed by Equation (1).

$$
W_{i j}=\exp \left[-d\left(x_{i}, x_{j}\right) / 2 \sigma^{2}\right]
$$

where, $x_{i}$ and $x_{j}$ denoted the mean of the superpixels in the $\mathrm{L}^{*} \mathrm{~A} * \mathrm{~B} *$ color space; $\sigma^{2}$ was a constant that controlled the strength of the weight.

5) Given $\boldsymbol{G}$, the degree matrix was $\boldsymbol{D}=\operatorname{diag}\left\{d_{11}, \ldots, d_{n n}\right\}$, where $d_{i i}=\sum_{j} w_{i j}$. The resulted ranking function can be written as:

$$
f^{*}(i)=(D-\alpha W)^{-1} y
$$

where, $\alpha$ balanced the smooth constraints in the regularization function of manifold ranking algorithm.

The main steps of Manifold Ranking algorithm were summarized as follows:

Input: A dataset of image feature and required parameters

Step 1: Define a graph $\boldsymbol{G}$ on the input dataset, where the nodes were the dataset and the edges were connections between two K-Nearest Neighbour points

Step 2: Structure weight matrix $\boldsymbol{W}$, in which $w_{i j}$ was computed by Equation (1) if two nodes were connected, and $w_{i j}=0$ otherwise.

Step 3: From the weight matrix $\boldsymbol{W}$, compute degree matrix $\boldsymbol{D}$ which was a diagonal matrix.

Step 4: Compute the sum of the relevance of the $i^{\text {th }}$ node to all the queries by Equation (2).

Output: A matrix, denoting the relevance of each nodes to queries.

(2) Saliency detection of green apples

Based on the attention theories of early works for visual saliency, in this research, the nodes on the image boundary were used as background seeds, i.e., the labeled data (query samples) to rank the relevance of all the other regions. Taking top image boundary as an example, the nodes on this side were used as the queries and other nodes as the unlabeled data. Thus, the indicator vector $\boldsymbol{y}$ was given, and all the nodes were ranked based on Equation (2). Its complement was the saliency measure. The vector was normalized to the range between 0 and 1 , and the saliency map using the top boundary prior, $S_{t}$ can be written as:

$$
S_{t}(i)=1-\bar{f}^{*}(i) \quad i=1,2 ; \cdot N,
$$

where, $i$ was the index of a superpixel node on graph; $\bar{f}^{*}$ denoted the normalized vector.

Each element in $\boldsymbol{S}_{t}$ can also be viewed as the sum of the relevance of the node to all the queries. Similarly, the other three maps $S_{b}, S_{l}$ and $S_{r}$ were also computed using the bottom, left and right image boundary as queries. It was noted that the saliency maps were computed with different indicator vector $\boldsymbol{y}$ while the weight matrix $\boldsymbol{W}$ and the degree matrix $\boldsymbol{D}$ were fixed. That is, it needed to compute the inverse of the matrix $(\boldsymbol{D}-\boldsymbol{W})$ only once for each image. Since the number of superpixels was small, the matrix inverse in Equation (2) could be computed efficiently. Thus, the overall computational load for the four maps was low. The four saliency maps were integrated by the following process:

$$
S_{b q}(i)=S_{t}(i) \times S_{b}(i) \times S_{l}(i) \times S_{r}(i)
$$

By integration of four saliency maps, the effects of imprecise queries can be reduced. Some salient parts of object were identified (although the object was not entire), and it was facilitated selecting the nodes of the foreground salient objects.

While most regions of the salient objects were highlighted in $\boldsymbol{S}_{\boldsymbol{b q}}$, some background nodes were not adequately suppressed. To alleviate this problem and improved the results, the saliency maps were further improved. The saliency map of the first stage was binary segmented using an adaptive threshold. The foreground queries were used to rank other nodes and develop the final result as follows:

$$
S_{f q}(i)=\bar{f}^{*}(i)
$$

The main steps of the proposed salient object detection algorithm based on manifold ranking were summarized as follows:

Input: An image and required parameters

1) Segment the input image into superpixels, construct a graph $\boldsymbol{G}$ with superpixels as nodes, and compute its degree matrix $\boldsymbol{D}$ and 
weight matrix $\boldsymbol{W}$ by Equation (1).

2) Compute $(\boldsymbol{D}-\boldsymbol{\alpha} \boldsymbol{W})^{-1}$ and set its diagonal elements to 0 .

3) Form indicator vectors $\boldsymbol{y}$ with nodes on each side of image as queries, and compute their corresponding side-specific maps by Equation (2) and Equation (3). Then, compute the saliency map $S_{b q}$ by Equation (4).

4) Binarize $S_{b q}$ to form salient foreground queries and an indicator vector $\boldsymbol{y}$. Compute the saliency map by Equation (5) .

Output: A saliency map $S_{f q}$ representing the saliency value of each superpixel.

\subsubsection{Gaussian curve fitting}

In this section, the images of silent region were transformed from RGB color space into YUV space. The $V$-component can almost distinguish the fruit and background (Figure 1b). It can be calculated by Equation (6):

$$
\boldsymbol{V}=\boldsymbol{R}-\boldsymbol{Y}
$$

where, $\boldsymbol{R}$ is the red component in RGB color space and $\boldsymbol{Y}$ represents luminance in YUV space. So the value of $\boldsymbol{V}$ was low and had no relation with luminance of images.

It was subject to Gaussian distribution approximately ${ }^{[21]}$ (Figure 1c). And appropriate segmentation threshold could be used to find the lost foreground pixels based on Gaussian curve fitting.

Given the model function of Gaussian distribution as:

$$
y=\frac{1}{\sqrt{2 \pi} \sigma} \mathrm{e}^{\frac{(x-\mu)^{2}}{2 \sigma^{2}}}
$$

where, $\mu$ is the mean which is a location parameter; $\sigma$ is the standard deviation and influences the degree of dispersion of distribution curve.

According to Pauta criterion:

$$
P\{|x-\mu|<3 \sigma\}=99.74 \%
$$

where, $\mu$ is the mean or expectation of the distribution (and also its median and mode), $\delta$ is the standard deviation.

Selecting the segmentation threshold $T$ that included foreground in $[u-3 \delta, u+3 \delta]$ can binarize the collected images adaptively.

The main steps of the proposed threshold selection based on Gaussian curve fitting were summarized as follows:

Step 1: Transform the result image of salient object detection algorithm into YUV color space, and apply Gaussian curve fitting to fit the $V$-component distribution (Equation (7)).

Step 2: Choose segmentation threshold T by Equation (8).

Step 3: Segment the image by $T$.

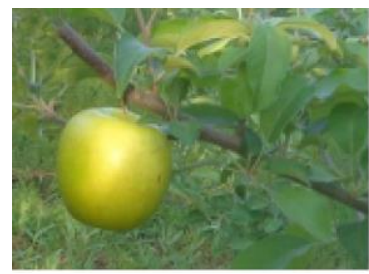

a. Original image

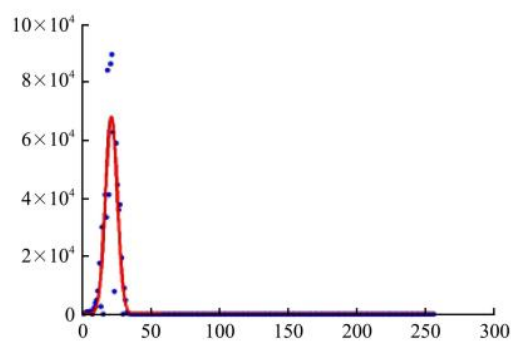

c. $V$-component distribution map

Figure 1 Original image and images about its $V$-component

\subsubsection{Evaluation metrics}

Four criteria including recognition ratio (by Equation (9)), false detection rate (FDR by Equation (10)), false positive rate (FPR by Equation (11)) and false negative rate (FNR by Equation (12)) were used to evaluate the segmentation results as quantitative analysis.

Recognition Ratio $=\left(B W \cap B W_{\text {real }}\right) / B W_{\text {real }} \times 100 \%$

Segmentation Error $=\left|B W_{\text {real }}-B W\right| / B W_{\text {real }} \times 100 \%$

False Positive Rate $=\left|B W-B W \cap B W_{\text {real }}\right| / B W_{c} \times 100 \%$

False Negative Rate $=\left|B W_{\text {real }}-B W \cap B W_{\text {real }}\right| / B W_{\text {real }} \times 100 \%$ (12)

where, $B W_{\text {real }}$ is the pixels of apple object extracted artificially, $B W$ is the pixels of apple object detected by proposed algorithm and $B W_{c}$ is the complementary set of $B W_{\text {real }}$. The effect of segmentation is better when FPR, FNR, and recognition ratio are higher, and FDR is lower.

\section{Results and discussion}

\subsection{Results}

Results of $k$-means algorithm and FCM algorithm were shown in Figure 2 and Figure 3 respectively. The parts of apple contour were lost, and a lot of leaves and other region pixels were in apple sub-image (Figure $2 b$ and Figure $3 b$ ).

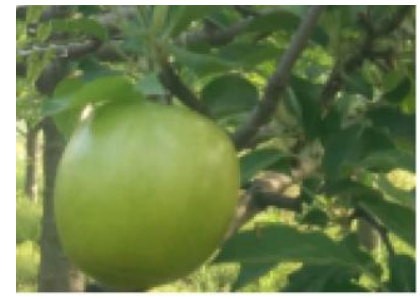

a. Original image

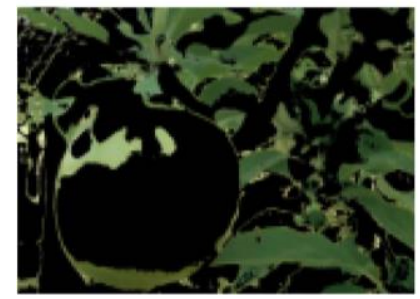

c. Leaf sub-image

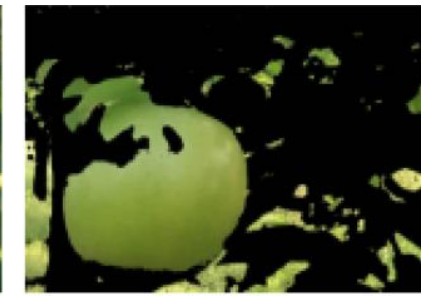

b. Apple sub-image

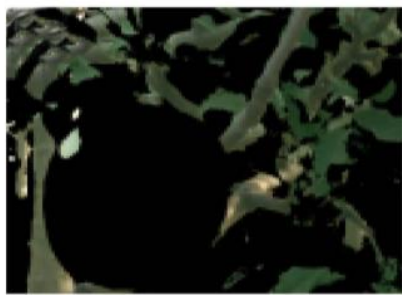

d. Other region sub-image

Figure 2 Original image and its processing results of $k$-means clustering algorithm

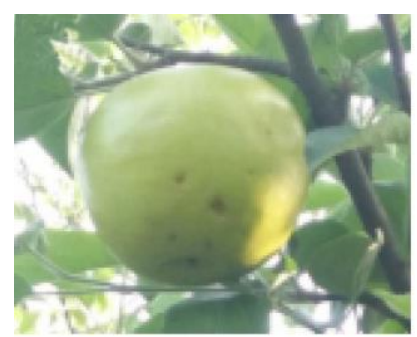

a. Original image

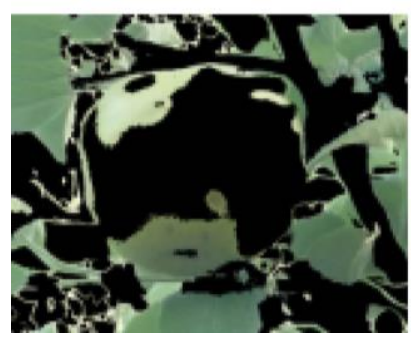

c. Leaf sub-image



b. Apple sub-image

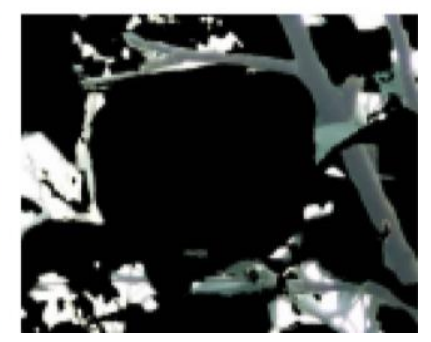

d. Other region sub-image

Figure 3 Original image and its processing results of FCM clustering algorithm 
Results of saliency detection were shown in Figure 4. The two parameters were empirically chosen, $\sigma^{2}=0.1$ and $\alpha=0.99$, for all the experiments. Figure $4 \mathrm{a}$ showed the input image, it was observed that the color of object was similar to backgrounds. It is hard to recognize the target by conventional method. Figure $4 \mathrm{~b}$ showed the image of $S_{b q}$. While most regions of the salient objects were highlighted in the first stage, some foreground nodes were suppressed. To alleviate this problem and improve the results, the saliency maps were further improved via ranking with foreground queries to generate $S_{f q}$. Figure $4 \mathrm{c}$ showed the image of $S_{f q}$. And the object in Figure $4 \mathrm{c}$ is more complete. Bi-segment

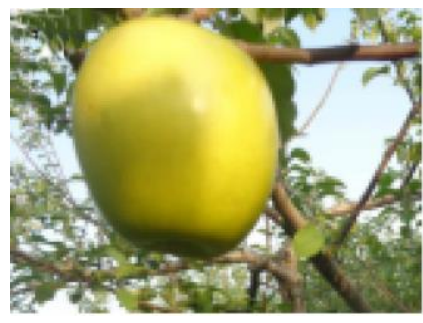

a. Original image

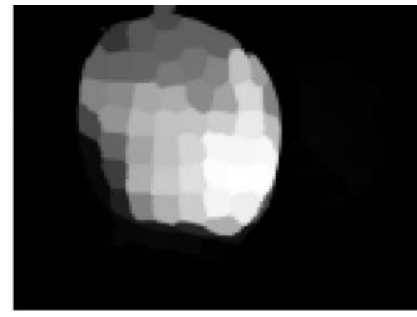

b. Image of $S_{b q}$

$S_{f q}$, the salient region was obtained as shown in Figure 4d.

Figure $5 \mathrm{~b}$ was the result of saliency detection and the object was not integrated. As shown in Figure 5c, there were also some pixels of the green apple lost in non-salient region and the saliency detection should be improved by Gaussian curve fitting. Comparing Figure 5b with Figure 5d, it was found that the latter one was more accurate after Gaussian curve fitting. However, there were more background pixels in it, the reason is that the distribution of $V$-component was dense and it was inevitable to cause error when chose a threshold. But, they were isolated and can be removed easily.

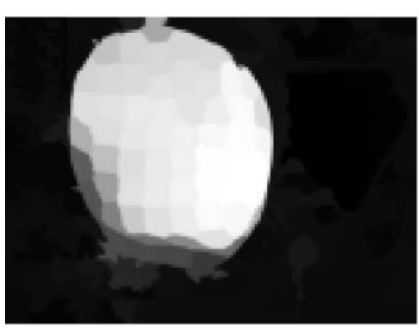

c. Image of $S_{f q}$

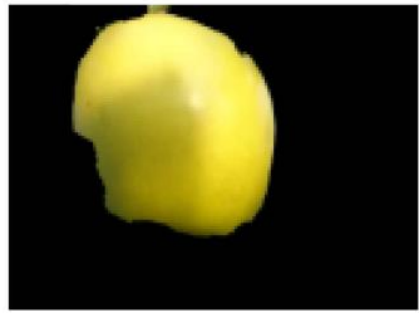

d. Salient region sub-image

Figure 4 Original image and its processing results of saliency detection via graph-based manifold ranking

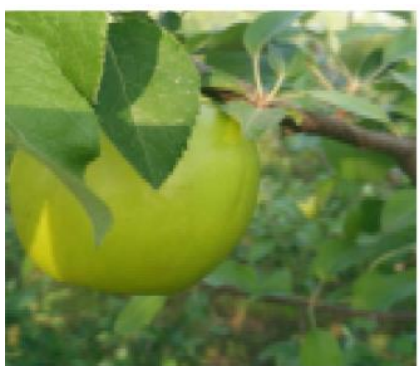

a. Original image

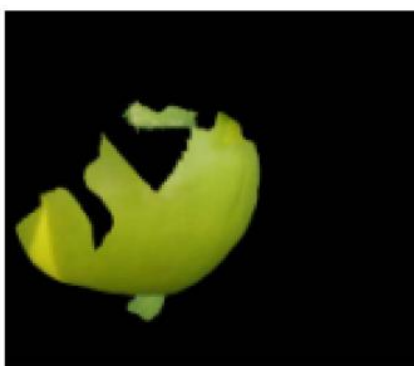

b. Salient region sub-image

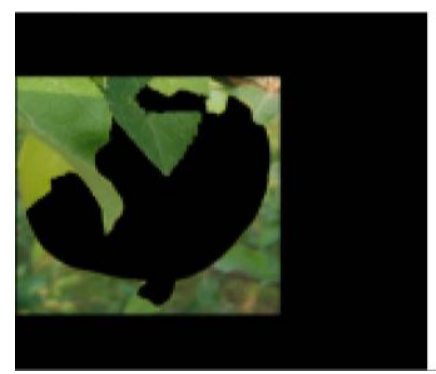

c. Complement of salient region sub-image

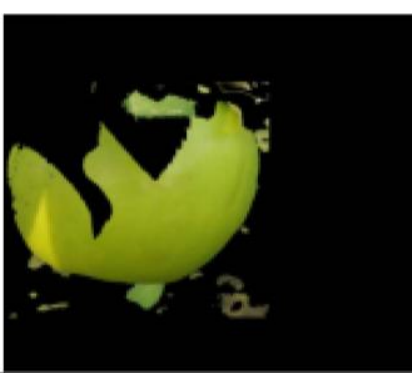

d. Result of the complete operation

Figure 5 Original image and its processing results of Gaussian curve fitting operation

From top to bottom in Figure 6, they were image where fruits were overlapping, image that had more than one separated fruits, image in which fruit was overlapped by leaves or branches, image in backlighting condition and image in frontlighting condition. This showed that the proposed method can get accurate results in the vast majority of nature scenes. There were two apples in original image in the second row, however only one apple was detected in the result of proposed method. It is because the smaller one was less salient than the bigger one and was seen as backgrounds. It can be inferred that the green apple can be segmented effectively, and the integrity of target can be guaranteed in terms of color and shape. Also, the results provided a reference for researching on the automatic recognition of green apples.

The average recognition ratio of the proposed algorithms was $91.84 \%$, which was higher than saliency detection algorithm, $k$-means clustering algorithm and FCM by $2.65 \%, 19.82 \%$ and $19.63 \%$, the average FDR was $4.22 \%$, which was higher than saliency detection algorithm by $1.69 \%$, lower than $k$-means clustering algorithm and FCM by $4.22 \%$ and $4.95 \%$, the average FNR was $8.16 \%$, which was lower than saliency detection algorithm, $k$-means clustering algorithm and FCM by $2.65 \%$, $19.82 \%$ and $19.63 \%$, the average FPR was $1.36 \%$, which was higher than saliency detection algorithm by $0.50 \%$, lower than $k$-means clustering algorithm and FCM by $0.82 \%$ and $1.12 \%$, respectively.

The standard deviations of proposed method and saliency detection were lower than those of $k$-means clustering algorithm and FCM clustering algorithm (Figure 8), which indicated that the saliency detection and proposed method were more steady in recognizing the green apples.

\subsection{Discussion}

In order to implement the complete segmentation of green apple, the saliency algorithm via graph-based manifold ranking was used to detect the object, while the results did not always contain the whole object, so we applied the Gaussian curve fitting method to supplement the loss area and achieved the integrity of segmentation results. The results were more accurate.

The FNR of sample 2 was large (Table 1) which implied that a larger region of target was assigned to background. It was mainly because of the existence of edges with shadows. And the proposed method had some difficulties in assigning these pixels correctly. As shown in Figure 4, the right edge and bottom edge of apple were shaded, and had high consistency with the surrounding, so the segmentation effect was not good enough. It still needs further research.

The FPR of sample 5 was large (Table 1) which implied that a larger region of background was assigned to object region. The facula around apples was of great difference with background in distance space and color space (Figure 9a), so the nodes of facula were selected as foreground queries (Figure 9b) and these superpixels cannot be removed in $S_{f q}$ (Figure 9c). The result image contained a lot of background pixels which resulted in the large FPR (Figure 9d).

These two parameters $\sigma^{2}$ and $\alpha$ were chosen empirically for all the experiments. It is difficult to use a property value for each special image. And this may lead to inaccurate results to some extent. 

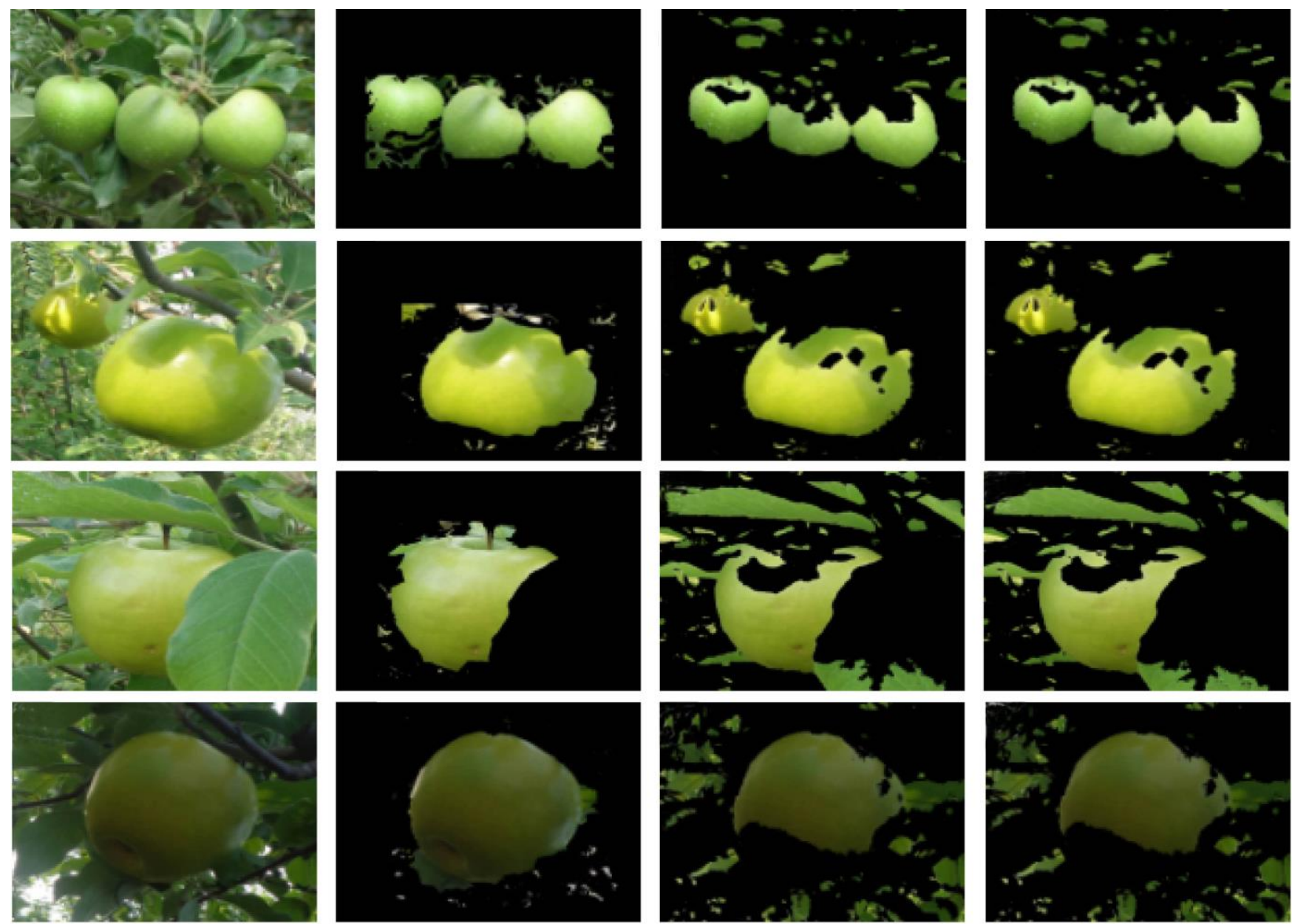

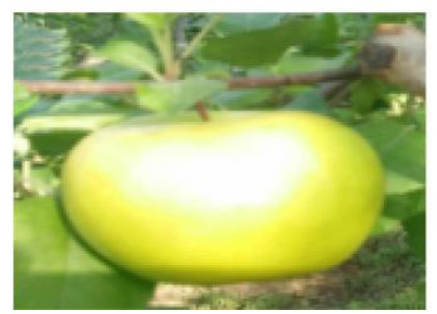

a. Original images

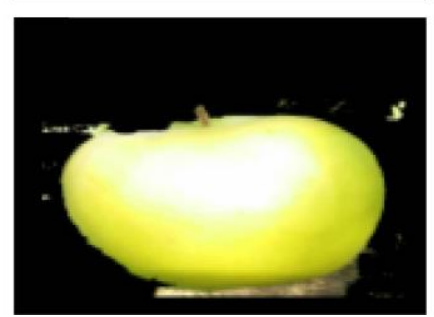

b. Results of proposed method

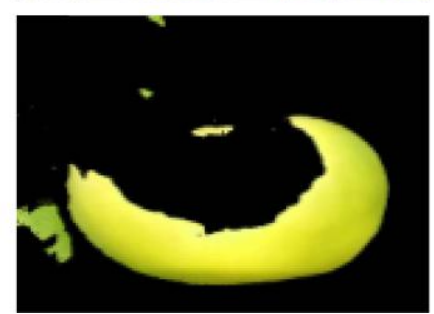

c. Results of $k$-means clustering algorithm

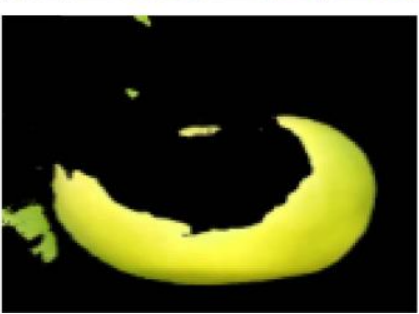

d. Results of FCM

Figure 6 Examples of green apple targets segmentation results

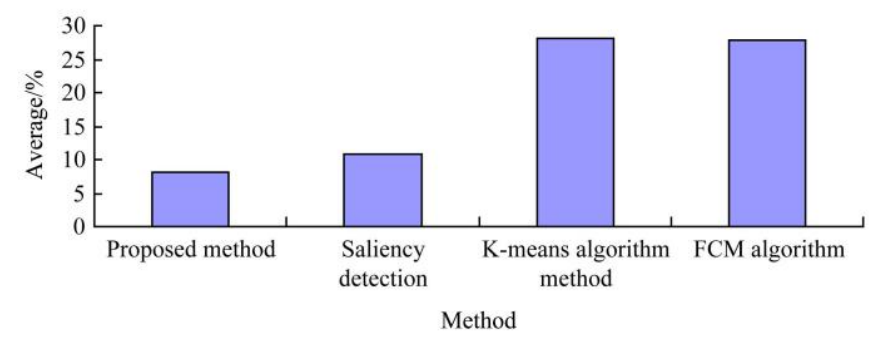

a. Histogram of FNR

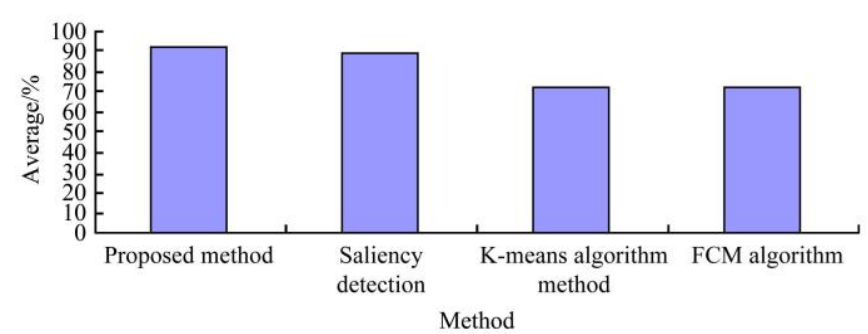

c. Histogram of Recognition Ratio

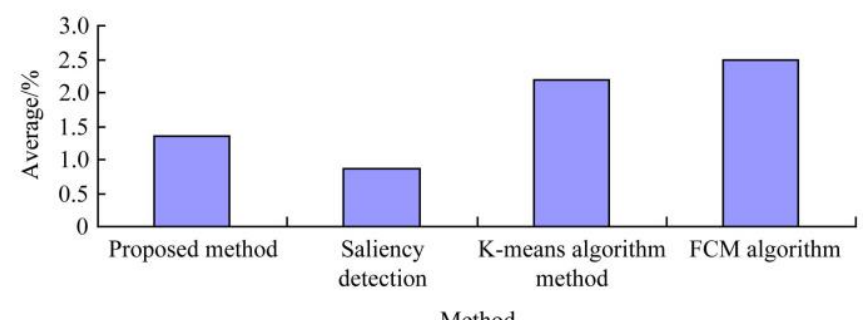

b. Histogram of FPR



d. Histogram of FDR

Figure 7 Histogram of four metrics average 


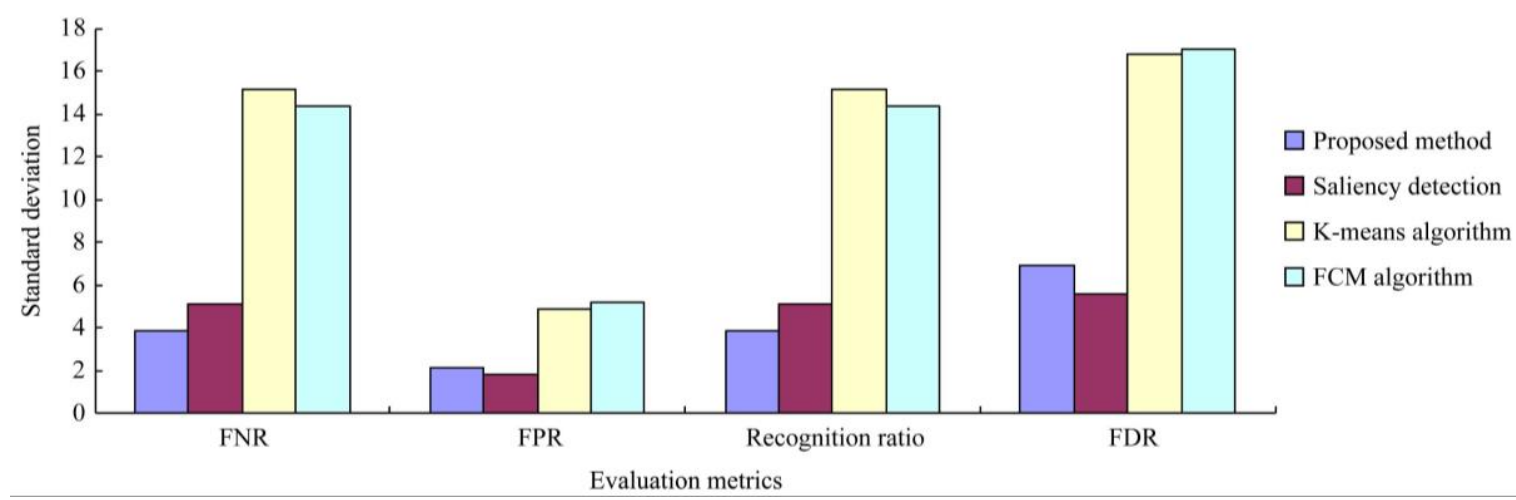

Figure 8 Histogram of four method standard deviation

Table 1 Evaluation index statistic of different segmentation methods

\begin{tabular}{|c|c|c|c|c|c|c|c|c|c|c|c|c|c|c|c|c|}
\hline \multirow{2}{*}{ No. } & \multicolumn{4}{|c|}{ FNR/\% } & \multicolumn{4}{|c|}{ FPR/\% } & \multicolumn{4}{|c|}{ Recognition Ratio/\% } & \multicolumn{4}{|c|}{ FDR/\% } \\
\hline & $\begin{array}{c}\text { Proposed } \\
\text { method }\end{array}$ & $\begin{array}{l}\text { Saliency } \\
\text { detection }\end{array}$ & $k$-means & FCM & $\begin{array}{c}\text { Proposed } \\
\text { method }\end{array}$ & $\begin{array}{l}\text { Saliency } \\
\text { detection }\end{array}$ & $k$-means & FCM & $\begin{array}{c}\text { Proposed } \\
\text { method }\end{array}$ & $\begin{array}{l}\text { Saliency } \\
\text { detection }\end{array}$ & $k$-means & $\mathrm{FCM}$ & $\begin{array}{c}\text { Proposed } \\
\text { method }\end{array}$ & $\begin{array}{l}\text { Saliency } \\
\text { detection }\end{array}$ & $k$-means & FCM \\
\hline 1 & 6.85 & 8.27 & 14.1 & 14.28 & 1.01 & 0.96 & 9.82 & 9.81 & 93.15 & 91.73 & 85.9 & 85.72 & 4.08 & 3.92 & 39.96 & 39.93 \\
\hline 2 & 14.48 & 14.67 & 41.14 & 42.09 & 0.17 & 0.09 & 4.66 & 4.63 & 85.52 & 85.33 & 58.86 & 57.91 & 0.25 & 0.13 & 7.14 & 7.09 \\
\hline 3 & 7.79 & 12.49 & 17.93 & 17.96 & 2.78 & 0.25 & 0.13 & 0.13 & 92.21 & 87.51 & 82.07 & 82.04 & 5.94 & 0.53 & 0.29 & 0.29 \\
\hline 4 & 7.64 & 7.49 & 29.2 & 28.42 & 0.03 & 0.03 & 3.98 & 4.21 & 92.36 & 92.51 & 70.8 & 71.58 & 0.11 & 0.1 & 14.34 & 15.15 \\
\hline 5 & 6.55 & 6.41 & 12.17 & 11.9 & 3.36 & 3.32 & 9.23 & 9.21 & 93.45 & 93.59 & 87.83 & 88.1 & 21.76 & 21.63 & 60.19 & 60.04 \\
\hline 6 & 10.92 & 11.39 & 28.32 & 43.08 & 0.69 & 0.64 & 0.46 & 0.2 & 89.08 & 88.61 & 71.68 & 56.92 & 1.36 & 1.29 & 0.92 & 0.4 \\
\hline 7 & 5.87 & 6.08 & 69.66 & 70.43 & 0.57 & 0.29 & 9.27 & 9.34 & 94.13 & 93.92 & 30.34 & 29.57 & 1.46 & 0.77 & 23.99 & 24.19 \\
\hline 8 & 7.82 & 8.94 & 22.35 & 24.29 & 0.06 & 0.02 & 0.05 & 0.03 & 92.18 & 91.06 & 77.65 & 75.71 & 0.11 & 0.04 & 0.09 & 0.05 \\
\hline 9 & 9.55 & 9.65 & 32.16 & 31.46 & 6.28 & 6.21 & 0.01 & 0.01 & 90.45 & 90.35 & 67.84 & 68.54 & 14 & 13.89 & 0.03 & 0.03 \\
\hline 10 & 5.03 & 4.9 & 7.86 & 8.37 & 0.08 & 0.07 & 1.57 & 1.56 & 94.97 & 95.1 & 92.14 & 91.63 & 0.8 & 0.73 & 16.29 & 16.32 \\
\hline 11 & 12 & 12.49 & 16.98 & 17.1 & 0.65 & 0.44 & 0.07 & 0.06 & 88 & 87.51 & 83.02 & 82.9 & 2.12 & 1.43 & 0.21 & 0.21 \\
\hline 13 & 10.7 & 10.66 & 48.92 & 42.2 & 2.06 & 1.81 & 4.24 & 5.07 & 89.3 & 89.34 & 51.08 & 57.8 & 5.22 & 4.6 & 10.77 & 12.93 \\
\hline 14 & 4.18 & 4.28 & 20.17 & 20.19 & 0.53 & 0.5 & 0.04 & 0.05 & 95.82 & 95.72 & 79.83 & 79.81 & 1.89 & 1.8 & 0.14 & 0.18 \\
\hline 15 & 8.21 & 11.4 & 5.18 & 5.44 & 0.55 & 0.5 & 4.42 & 4.31 & 91.79 & 88.6 & 94.82 & 94.56 & 2.41 & 2.17 & 19.27 & 18.76 \\
\hline 16 & 6.79 & 8.09 & 12.32 & 12.14 & 0.64 & 0.55 & 2.09 & 2.13 & 93.21 & 91.91 & 87.68 & 87.86 & 1.34 & 1.15 & 4.36 & 4.45 \\
\hline 17 & 5.9 & 10.84 & 15.4 & 15.16 & 0.2 & 0.04 & 1.07 & 1.09 & 94.1 & 89.16 & 84.6 & 84.84 & 1.61 & 0.33 & 8.78 & 8.89 \\
\hline 18 & 5.18 & 5.07 & 43.26 & 44.17 & 0.51 & 0.49 & 20.84 & 20.46 & 94.82 & 94.93 & 56.74 & 55.83 & 1.24 & 1.19 & 50.7 & 49.75 \\
\hline 19 & 13.32 & 13.2 & 25.57 & 27.14 & 0.03 & 0.02 & 25.56 & 22.99 & 86.68 & 86.8 & 74.43 & 72.86 & 0.09 & 0.08 & 81 & 72.89 \\
\hline ...... & $\ldots \ldots$ & $\ldots \ldots$ & & $\ldots \ldots$ & $\ldots \ldots$ & $\ldots \ldots$ & $\ldots \ldots$ & $\ldots \ldots$ & $\ldots$ & $\ldots \ldots$ & $\ldots \ldots$ & $\ldots \ldots$ & $\ldots \ldots$ & $\ldots \ldots$ & $\cdots$ & ...... \\
\hline 55 & 4.58 & 7.14 & 49.18 & 39.07 & 0.08 & 0 & 1.59 & 1.78 & 95.42 & 92.86 & 50.82 & 60.93 & 0.94 & 0.02 & 18.75 & 20.88 \\
\hline ave & 8.16 & 10.81 & 27.98 & 27.79 & 1.36 & 0.86 & 2.18 & 2.48 & 91.84 & 89.19 & 72.02 & 72.21 & 4.22 & 2.53 & 8.44 & 9.17 \\
\hline STD & 3.88 & 5.15 & 15.12 & 14.33 & 2.15 & 1.83 & 4.85 & 5.2 & 3.88 & 5.15 & 15.12 & 14.33 & 6.92 & 5.61 & 16.82 & 17.02 \\
\hline
\end{tabular}

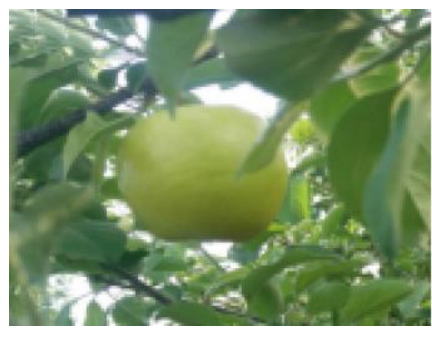

a. Original image

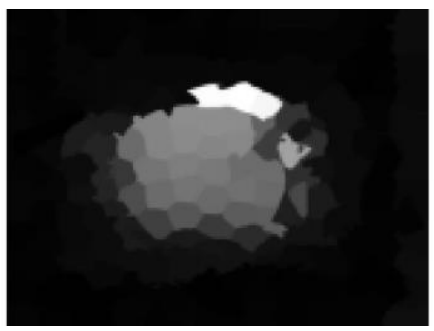

b. Image of $S_{b q}$

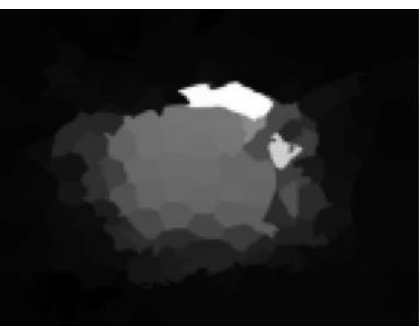

c. Image of $\boldsymbol{S}_{f q}$

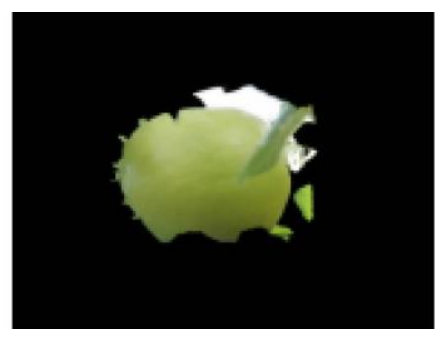

d. Salient region sub-image

Figure 9 Original image and its processing results of saliency detection via graph-based manifold ranking

\section{Conclusions}

According to the characteristics of apple fruit, this paper proposed a detection algorithm of green fruits based on saliency theory and Gaussian curve fitting, and realized the adaptive segmentation of apple object under the natural scenes. Main conclusions are as follows:

(1) Detecting the salient region of image and then searching the lost target by Gaussian distribution theory can effectively improve the recognition rate of apple target under the natural scene. After testing on multiple images, the results show that the recognition rate is $91.84 \%$ on average, and this method is effective and feasible in detecting apple object.

(2) The method has poor performance in segmentation of edges with shadows and intense facula around the object. The future work will focus on these problems, and the selection of parameters for each image. 


\section{Acknowledgments}

This study was supported by the National High Technology Research and Development Program of China ("863" Program) (No. 2013AA10230402), Agricultural science and technology project of Shaanxi Province (No. 2016NY-157) and Fundamental Research Funds Central Universities (2452016077). The authors would like to thank all the authors cited in this article and anonymous referees for their helpful comments and suggestions.

\section{[References]}

[1] Wang Y D, Zhang X Z. Segmentation algorithm of muskmelon fruit with complex background. Transactions of the CSAE, 2014; 30(2): 176-181. (in Chinese)

[2] Ji W, Meng X L, Tao Y, Xu B, Zhao D A. Fast segmentation of colour apple image under all-weather natural conditions for vision recognition of picking robots. International Journal of Advanced Robotic Systems, 2016 13(1): 24-32

[3] Luo L F, Zou X J, Xiong J T, Zhang Y, Peng H X, Lin G H. Automatic positioning for picking point of grape picking robot in natural environment. Transactions of the CSAE, 2015; 31(2): 14-21. (in Chinese)

[4] Chen Y L, Park S-K, Ma Y D, Ala R. A new automatic parameter setting method of a simplified PCNN for image segmentation. IEEE Transactions on Neural Networks, 2011; 22(6): 880-892.

[5] Jia W K, Zhao D A, Liu X Y, Tang S P, Ruan C Z, Ji W. Apple recognition based on k-means and GA-RBF-LMS neural network applicated in harvesting robot. Transactions of the CSAE, 2015; 31(18): 175-183. (in Chinese)

[6] Xu L M, Lv J D. Bayberry image segmentation based on homomorphic filtering and k-means clustering algorithm. Transactions of the CSAE, 2015; 31(14): 202-208. (in Chinese)

[7] Peng H X, Zou X J, Chen Y, Yang L, Xiong J T, Chen Y. Fruit image segmentation based on evolutionary algorithm. Transactions of the CSAE 2014; 30(18): 294-301. (in Chinese)

[8] Cheng M M, Mitra N J, Huang X L, Torr P H S, Hu S M. Salient object detection and segmentation. IEEE Transactions on Pattern Analysis \& Machine Intelligence, 2013; 37(3): 1
[9] Rutishauser U, Walther D, Koch C, Perona P. Is bottom-up attention useful for object recognition? IEEE Computer Society Conference on Computer Vision \& Pattern Recognition, 2004; 2: II-37- II-44.

[10] Itti L. Automatic foveation for video compression using a neurobiological model of visual attention. IEEE Transactions on Image Processing: A Publication of the IEEE Signal Processing Society, 2004; 13 : 1304-1318.

[11] Chen T, Cheng M M, Tan P, Shamir A, Hu S M. Sketch2Photo: internet image montage. Acm Transactions on Graphics, 2009; 28: 89-97.

[12] Liu T, Sun J, Zheng N N, Tang X, Shum H Y. Learning to detect a salient object. IEEE Transactions on Pattern Analysis \& Machine Intelligence, $2011 ; 33$ : 353-367.

[13] Harel J, Koch C, Perona P. Graph-based visual saliency. Advances in Neural Information Processing Systems, 2006; 19: 545-552.

[14] Sun J, Lu H, Li S. Saliency detection based on integration of boundary and soft-segmentation. 19th IEEE International Conference on Image Processing (ICIP), 2012; pp.1085-1088.

[15] Lu Y, Zhang W, Lu H, Xue X. Salient object detection using concavity context. IEEE International Conference on Computer Vision, 2011; pp.233-240.

[16] Cheng M, Mitra N J, Huang X, Torr P H S, Hu S. Global contrast based salient region detection. IEEE Conference on Computer Vision and Pattern Recognition, 2011; pp.409-416.

[17] Xie Y L, Lu H C, Yang M-H. Bayesian saliency via low and mid level cues. IEEE Transactions on Image Processing A Publication of the IEEE Signal Processing Society, 2013; 22(5): 1689-1698.

[18] Yang C, Zhang L, Lu H, Xiang R, Yang M H. Saliency detection via graph-based manifold ranking. IEEE Conference on Computer Vision and Pattern Recognition, 2013; pp.3166-3173.

[19] Arora P, Deepali Dr, Varshney S. Analysis of k-means and $k$-medoids algorithm for big data. Procedia Computer Science, 2016; 78: 507-512.

[20] Khalid N E A, Noor N M, Ariff N M. Fuzzy c-means (FCM) for optic cup and disc segmentation with morphological operation. Procedia Computer Science, 2014; 42: 255-262.

[21] Biswas S, Ghoshal D, Hazra R. A new algorithm of image segmentation using curve fitting based higher order polynomial smoothing. Optik-International Journal for Light and Electron Optics, 2016; 127(20): 8916-8925. 